hospitalizations of persons with BE in Germany between 2005 and 2011, adjusted for age by 2.9\% per year. Similar data were recorded in the US: general prevalence of BE is unknown exactly, by the recent estimates - 51 cases per 100,000 people, most likely underestimated. In a recent multicenter European study involving 1310 patients with EB, the frequency of exacerbations was estimated to be 1.8-3.0 in 1 patient per year, with a follow-up hospitalization rate of 26.6$31.4 \%$ for 2 years of follow-up. In Russian Federation, there is no precise data on prevalence of BE. However, due to the improvement in lung imaging technology, the prevalence of BE should be revised. In the pathogenesis of BE, great importance is attached to two main factors - the local inflammatory (suppuration) process in the bronchi (local purulent endobronchitis) and obstructive atelectasis. Obturation bronchus and bronchial secretions delay excretion contribute to the development and progression of inflammation (suppurative) process in a bronchus lumen distal to the obstruction.

The pathophysiological mechanism of development of BE is most correctly understood in terms of the hypothesis of the "vicious circle": progression of the disease is associated with insufficient evacuation of sputum in the airways, bacterial colonization, inflammation of the respiratory tract and their structural damage. Therefore, the goal of therapy should be to stop or reverse these processes and thereby to "break the vicious circle".

The clinical observation of the patient S., 44 years old, who entered the pulmonology department of the Amur Regional Clinical Hospital with complaints of asthma attacks up to 6-8 times a day, including those that are nightly, dabbled with berodual, dyspnea at rest, cough with difficult to separate mucopurulent sputum, temperature raising to $37.8^{\circ} \mathrm{C}$. From anamnesis it is known that asthma attacks disturbing from 17 years of age, acute - up to 3-4 times a year, mainly in spring - autumn period. Allergic history burdened: polyvalent sensitization (household, pollen, food). In 2002 suffered bilateral pneumonia. Since 2005 he's taking systemic glucocorticoids (prednisolone) in a constant maintenance dose of $10 \mathrm{mg}$ per day, Symbicort, Berodual. Twice in the anamnesis (in 2010 and 2011) - life-threatening exacerbation of asthma, was hospitalized in the intensive care department.

The state of moderate severity, the auxiliary musculature participates in the act of breathing. Chest of emphysematous form, breathing weakened, dry wheezing wheezes on exhalation, BR - 28 per minute. The heart sounds are muffled, rhythmic, the accent of 2 tones on the pulmonary artery. According to CT of chest organs: cystic hypoplasia and bronchiectasis in the lower lobes of both lungs, the internal diameter of the bronchi is greater than the neighboring arteries, there is no typical form of bronchi (the same diameter as the parental branch> $2 \mathrm{~cm}$ ), the walls of the bronchi are thickened, thin-walled cystic formations, with horizontal levels of fluid levels. The conclusion of fibrobronchoscopy: signs of diffuse endobronchitis, intensity of inflammation III, hyperreactivity of bronchi II, a positive Sul sign. Subsequently, sanative bronchoscopies were performed. In the sprouting on microflora: pneumococcus $\mathrm{x} 106$, hemophilic rod x105, sensitive to amoxiclav, cefriaxone, levofloxacin.

Clinical diagnosis: Bronchial asthma, mixed form (atopic, infectious-dependent), severe uncontrolled course, steroid-dependent variant, exacerbation. COPD, mixed type, severe course, exacerbation. Cystic hypoplasia and bronchiectasis of the lower lobes of both lungs. $\mathrm{CPH}$, compensation stage. RI-II. Conclusions. This clinical case is interesting for the patient's comorbid state - the presence of steroid-dependent BA and COPD - the so-called cross-over syndrome - in combination with cystic hypoplasia and bronchiectasis, which dictates the need for a personified approach to choosing the amount of therapy to achieve asthma control and prevention of exacerbations.

\title{
References
}

1. Grigoriev E.G. Chronic festering lung diseases // Bulletin of the East Siberian Scientific Center of the Siberian Branch of the Russian Academy of Medical Sciences. 2014. № 4 (98). P.100-104.

2. Zarembo I.A., Kiseleva E.A., Zarayskaya L.S. and others. Bronchoectatic disease: a modern view of the problem // Practical pulmonology. 2015. № 4. P. 27-33.

3. Shoikhet J.N., Titova E.A., Dukov L.G. and others. Clinical course and antibacterial therapy of chronic obstructive pulmonary disease in combination with bronchiectasis // Siberian Medical Journal. 2013. T.28, №4. P.67-70.

UDC 616.34: 616.12-008 DOI 10.22448/AMJ.2017.3.142-143

\section{PECULIARITIES OF HEART RHYTHM DISTURBANCE IN CHILDREN WITH PATHOLOGY OF THE GASTROINTESTINAL TRACT}

\section{O.V. Shanova, E.F. Filonenko}

\section{Amur State Medical Academy, Blagoveshchensk, Russia}

Abstract: This article is devoted to the problem of arrhythmias in children with gastrointestinal pathology and their correlation with vegetative nervous system. A comparative assessment of arrhythmias in children with gastrointestinal pathology and without it. Children and adolescents with with gastrointestinal pathology have more wide range of cardiac arrhythmias and conduction failure. There is a group of changes associated with peculiarities of autonomic regulation (bradycardia, rhythm migration). These peculiarities require an integrated approach to the treatment of such patients with the use of vegetotrophic drugs.

Key words: children, gastrointestinal tract, heart rhythm disturbance.

Chronic pathology of the digestive tract in children is one of the topical problems of modern medicine. Extremely wide prevalence of the pathology of the gastroduodenal zone, the incidence of gastrointestinal tract (GI) diseases to the prolonged, recurrent course of frequent development of complications, a decrease in the quality of life of patients, economic losses pose the problem of fighting chronic gastroduodenal pathology in the category of so142 Амурский медицинский журнал №3 (19) 2017 
cial [1]. One of the possible causes of digestive tract diseases in children is the failure in the constantly growing body of regulatory systems and, above all, the autonomic nervous system [2]. Violations of vegetative homeostasis, leading to the disruption of the functions of organs and systems, contribute to a change in the level of metabolism and energy in the body and are an important pathogenetic link of many chronic diseases, including gastrointestinal ones[3]. It is assumed that the identification of features of the functioning of the autonomic nervous system and heart rate parameters will allow timely not only to diagnose, but also to correct their deviations [4].

The purpose of this study was to explore the features of changes in cardiac rhythm and conductivity, identified on an electrocardiogram (ECG), in children with chronic gastroduodenal pathology.

Materials and methods: The main group (I) consisted of 41 children aged 4 to 10 years (mean age 8,2) with chronic pathology of the gastroduodenal zone. Gender distribution was as follows: 18 boys (43,9\%) and 23 girls - (56,1\%). The control group (II) was 45 conditionally healthy children (without pathology of the gastrointestinal tract; from them - 18 girls (40\%) and 27 boys (60\%), the average age $-8,6$ years, groups were comparable by age and gender. The diagnosis was established on the basis of complaints, anamnesis of the disease, general clinical examination, data of fibroadastroduodenoscopy, ultrasound examination of the abdominal cavity organs, tests for the presence of Helicobacter pylori.

Results and discussion: The duration of the disease in the main group was as follows: 7 gastroduodenal pathology $(17,1 \%)$, duration of the disease 1 year - 14 people $(34,1 \%)$, duration of the disease over 1 year - 20 people $(48,8 \%)$; the average duration of the disease in children was 2,2 years. Diseases of the stomach and duodenum (gastritis, gastroduodenitis) predominated in the study group - 25 children (60,9\%), from them - 61,5\% was diagnosed erythematous gastritis, in $7 \%$ children - gastroesophageal reflux; diseases of the gallbladder and biliary tract (dysfunction of the biliary tract) - 3 patients (7,3\%); pancreas diseases (manifestations of dispensiveness, pancreatopathy) - 13 children (31,7\%). 39\% of children in first group had concomitant pathology: weight loss in 12,5\%. 1/3 children form this group had concomitant pathology: weight deficit was in 12,5\%, cardiac pathology - in 37,5\%, orthopedic pathology - in $18,7 \%$ and $14 \%$ of children had endocrine and neurological diseases. According to the results of ultrasound in the main group, changes in the pancreas (structural changes, densification of the Virsung duct) were found in $41,2 \%$ patients, changes in the liver (reactive changes in the liver parenchyma, compaction of the periportal structures) were detected in $32,3 \%$ and changes in the gallbladder (deformation, inflection) - in $26,5 \%$ children.

Having studied ECG data in the main group of changes, 95,1\% of children were registered, which is significantly more likely than in children of the control group 48,8\% ( $p<0.05)$. So the frequency of bradycardia was $53,6 \%$, which is significantly more likely than in group II $(20 \%)(p<0,05)$, which have indicated the vagal nature of these diseases. Ectopic rhythm and rhythm migration also occurred more often in patients of the first group, but without significant differences $(19,5 \%$ и $8,9 \%$ accordingly). Normocardia prevailed in the children of the control group (70\%) (p $<0,01)$. Violations of the conduction in the ventricles were significantly more frequent in children and adolescents with gastroduodenal pathology $(65,9 \%)$ than in the control group $(24.4 \%)(p<0,05)$. On average, one patient of the first group had 1,2 $\pm 0,1$ rhythm and conduction disorders and in $I I-0,6 \pm 0,2$ (2 times less) ( $p<0.05)$. In addition, the combination of arrhythmias and conduction disorders was noted only in the children of the main group (15,9\%).

Conclusions: Thus, ECG analysis indicates significant differences in the quantitative and qualitative nature of electrogenesis in children and adolescents in the main group. This allows us to distinguish a group of changes (in the form of bradycadias and conduction disorders) associated with the pathology of the gastrointestinal tract and the peculiarity of the functioning of regulatory systems. The high frequency of cardiac arrhythmias and conduction in gastroduodenal pathology indicates a considerable significance of these disorders in the genesis of the development of these conditions. When carrying out medical and recreational activities in children with chronic gastroduodenal diseases, along with standard methods of examination, it is necessary to study the condicion of the autonomic nervous system for more effective pathogenetic and symptomatic treatment.

\title{
References:
}

1. Suprun I.M., Makarova V.I. State of the autonomic nervous system in children with atopic dermatitis, with chronic diseases of the stomach and duodenum // Human Ecology - 2006. - № 12. - P. 22-26.

2. Sikorsky A.V. Symptomatic arterial hypotension in children with chronic gastroduodenal pathology: peculiarities of central hemodynamics and heart rhythm // Journal of GGMU. - 2003 - № 1. - P. 28-31.

3. Zanko S.N. Variability of heart rhythm and cerebral hemodynamics in children with chronic gastroduodenal pathology // Reviewed scientific and practical journal "Protection of motherhood and childhood". - 2005. - №1(6). - P. 17-23.

4. Chupak E.L. Features of vegetative reactivity in adolescents with bronchial asthma, taking into account the initial vegetative tone // Proceedings of the VII Congress of pulmonologists in Siberia and the Far East (with international participation).- Blagoveshchensk, 2017. P. 207-210.

UDC 616.24-002-005:616-008.9 DOI 10.22448/AMJ.2017.3.143-146

\section{PECULIARITIES OF CLINIC AND HEMODYNAMICS IN COMORBID COURSE OF OBSTRUCTIVE PULMONARY DISEASE AND METABOLIC SYNDROME}

\author{
O.A. Tanchenko, S.V. Naryshkina
}

FSBEI HE Amur State Medical Academy

Summary: Chronic obstructive pulmonary disease (COPD) belongs to a group of chronic inflammatory lung diseases characterized by a partially reversible airflow limitation. Among the numerous comorbid conditions of COPD, in more detail we examined the role of factors of systemic inflammation, arterial stiffness, carbohydrate and lipid metabolism in the progression of the comorbid course of COPD and the main components of the metabolic syndrome. It was revealed that an increase of arterial stiffness and systemic inflammation appear to be an indepen- 